\title{
Retraction Note to: Non-relativistic analytical solutions of the Kratzer potential for a perturbed system in a magnetic field
}

\section{H. F. Kisoglu ${ }^{a}$}

Department of Basic Sciences, Faculty of Maritime, Mersin University, Mersin, Turkey

(C) The Author(s), under exclusive licence to Società Italiana di Fisica and Springer-Verlag GmbH Germany, part of Springer Nature 2021

\section{Retraction Note: Eur. Phys. J. Plus (2019) 134: 460 https://doi.org/10.1140/epjp/i2019-12970-9}

The Editor-in-Chief retracted this article, [Eur. Phys. J. Plus (2019) 134: 460], as an outcome of an editorial investigation involving the results of Sect. 2, more specifically the results obtained in the case $\mathrm{N}_{3} 3$, and as reported in Tables 3, 4, and 5. The Author does not agree with this retraction. 